\title{
THE NATURE OF THE VARIABILITY OF DUST CONCENTRATIONS AT THE COAL FACE
}

\author{
BY \\ P. D. OLDHAM
}

From the Pneumoconiosis Research Unit of the Medical Research Council, Llandough Hospital, near Cardiff

(RECEIVED FOR PUBLICATION AUGUST 4, 1953)

It is well known that dust conditions in a coalmine vary from place to place and from time to time. Watson (1949) pointed out that even at one position on a long-wall face the concentration fluctuates wildly from minute to minute and varies even more from day to day, while the two halves of a double-unit face might differ considerably. Bedford and Warner (1943), when considering the concentration of dust in different mining operations, also noted that there was great variation in the individual dust counts and in addition large variations from mine to mine, so much so that it was not practicable to estimate with any reasonable accuracy an average dust concentration for each mining operation. Other difficulties which this variation causes in dust sampling work have been emphasized by several writers, and Oldham and Roach (1952) have pointed out that it must be given due consideration in designing a sampling scheme which is efficient in terms of the effort needed to obtain a specified amount of information. Fortunately it appears that momentary variation is of little importance in the aetiology of pneumoconiosis. The arguments of Wright (1953) and Long (1953) show that an estimate of average dustiness and the period during which it operates is all that is required, an estimate which could most economically be obtained by means of automatic, long-term sampling instruments. However, so long as short-term, intermittent sampling instruments continue to be used, the effect of this variation must be considered before deductions about average dust conditions may safely be drawn. Equally, it must be allowed for in interpreting the records of past dust conditions. Accordingly, this paper will present some of the evidence that we possess at this Unit of its nature and magnitude, and will draw some practical conclusions.

In the past six years extensive sampling with the thermal precipitator has been carried out by teams from this Unit. The number of samples obtained (some 8,000 ) is large, although already eclipsed by the number taken by the National Coal Board's sampling teams, which are making ever-increasing use of the thermal precipitator, an accurate though inconvenient and short-term dust-sampling instrument. However, the samples obtained by this Unit have been concentrated at a relatively small number of mines, so that the number of samples representing each different environment is large. Mines with a big range of average concentrations were well represented. In consequence our data are particularly suited to the detection of any consistent patterns in the variation of concentration, such as might be obscured in data covering a less extensive range in less detail.

Few of the surveys we have carried out at this Unit have continued for more than two or three weeks, and it is clear that a statistical treatment of variation of concentration from day to day could not be satisfactory without a long series of daily samples. There are many factors determining the average level of dust conditions during a shift, some of them (such as the width of the workings from face to goaf) clearly likely to cause changes of a cyclic nature, others (the progress of a dust suppression programme, a change in the nature of the coal seam or surrounding strata) inducing more or less steady trends, while a large number will operate in an apparently random manner. Understanding of the effects of such factors will most readily be achieved by analysis of the results of routine dust sampling over long periods of time, provided that such sampling is carried out in accordance with sound statistical principles. This paper will be concerned only with the variation of concentrations about the average level during particular shifts at particular coal faces. 


\section{Material}

For the most part, our thermal precipitator sampling has been carried out at one or two fixed positions on long-wall faces, in particular at a position about 5 yards from the return end. Samples were also taken half way along the face, and occasionally at the intake end or in intermediate positions. Since the development of the " random collier" system of sampling (Oldham and Roach, 1952) samples have been taken at the working place of randomly chosen colliers, and thus, on the average, have been evenly distributed at all points of different faces. In earlier years the samples were taken systematically at regular intervals throughout the shift, though excluding the "snap" or midshift break. Subsequently, and always when the random-collier technique was in use, samples were taken at times chosen beforehand by means of random sampling numbers. In this case samples were taken during snap time if by chance the randomly chosen moment fell therein, but they have been excluded from this discussion. (It may be remarked that few samples taken during snap-time by this Unit have ever produced a countable deposit on the thermal precipitator cover-glasses; the concentrations in fact were almost always well below 100 particles $/ \mathrm{ml}$.) The data therefore concern the period during which work of any kind may be proceeding on the face, and exclude all regular periods of inactivity. The duration of each sample has been standardized of recent years at three minutes (equivalent to a $20 \mathrm{ml}$. volume of air), but originally ten-minute samples were occasionally taken.

In view of this diversity of procedure it is surprising that the variation of samples about the average for the shift is found to show high consistency and to be almost perfectly proportional to the value of the average. In Fig. 1 the samples obtained during approximately one in 10 of all the shifts sampled at any time by this Unit have been plotted, each at a position corresponding to the average for the shift. The concentrations are expressed as the number of particles between 0.5 and 5.0 microns per ml. of sampled air. The choice of shifts was made on a random basis, but with the intention of covering the whole range of mean concentrations more or less evenly. Accordingly results were taken from the Unit records in serial order, sets with means falling at a concentration already covered being rejected, until the requisite coverage was achieved. Samples from eight different collieries are included.

The scales of Fig. 1 are both logarithmic, so that the consistent width of the scatter of samples over the whole range of averages represents in fact a constancy of proportional variation. The majority of samples fall within a range of 30 to $250 \%$ of the shift mean.

The appearance of such proportional variation is characteristic of samples drawn from a population (in the statistical sense) which is of lognormal form. That is, the relative frequency of the logarithms of individual levels of dust concentration appears to be governed by the Normal law. So far as we are aware, this observation has not been previously made. It has been the custom in this Unit for some years to make a logarithmic transformation of dust-sampling results before making statistical tests of significance. The justification for this lay in the obvious increase of variability with intensity of concentration, which was likely to have invalidated the customary assumptions made about the form of sampling distributions. The transformation was observed to render the variability much more stable, but was thought to be merely a convenient device for this purpose. It now appears that not only are the logarithms of concentrations within a shift distributed quite closely in accordance with the Normal law, but that the shape of the Normal curve, determined by its standard deviation, is almost the same in a variety of different faces in different collieries.

It is, therefore, of great interest to estimate the standard deviation of this Normal distribution, for it appears that this parameter will be so nearly constant as to enable us to estimate with its aid the precision to be expected from any set of samples taken within a shift. Moreover, with a knowledge of the form of the distribution of concentrations within a shift, it will be easy to estimate, from the value of the mean concentration, or the total dust exposure measured by an automatic sampler, any other feature of the dust conditions. For example, those who believe that high concentrations are disproportionately dangerous compared with low or average concentrations may wish to test their hypothesis by relating the appearance of pneumoconiosis to the frequency of concentrations above some spesified level, a test which, without a knowledge of the relative frequency of high and average concentrations, would require a special sampling technique. As Long (1953) suggests, estimation of the average alone would be quite sufficient.

\section{Analysis}

In Fig. 1 samples obtained by the random-collier procedure are marked by crosses. It will be seen that they are fairly representative, except at low 
concentrations, of the complete set of results, and statistics calculated from them alone should adequately cover the complete data.

Three such surveys have been made by the Unit up to the present, two at one South Wales pit and one at a pit in England. The distribution of samples estimate, $0 \cdot 2203$, given in the fourth line of the table should adequately represent all three sets. The histogram in Fig. 2 was obtained by pooling the three sets of data and plotting the frequency of different deviations of individual samples from the shift mean, on a logarithmic scale. The normal

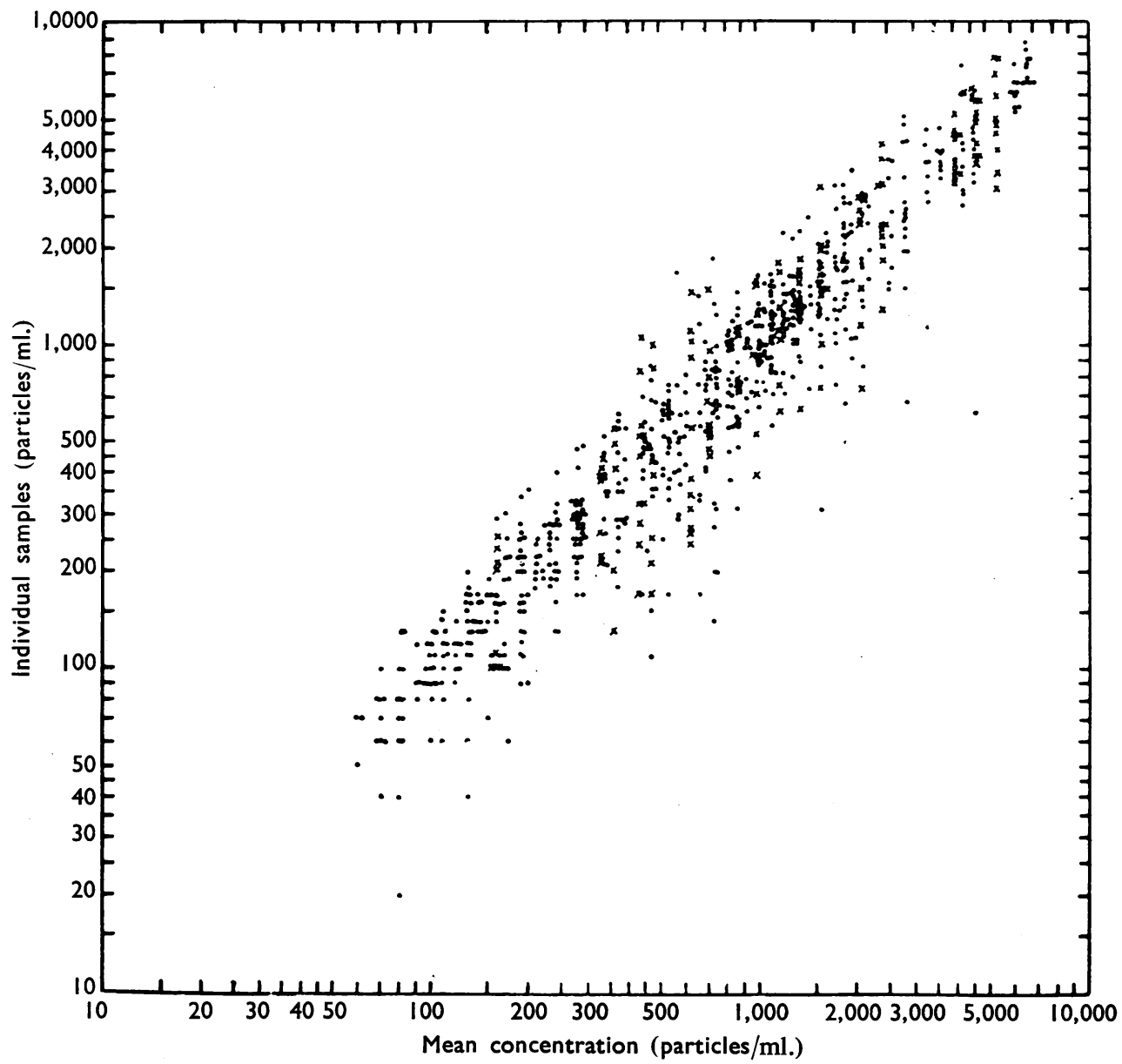

FIG. 1.-The relationship between individual samples and the estimated mean concentration of the shift on which they were obtained.

within a single shift (that is, within the stint of one random collier) is very similar in each survey. The standard deviations of these distributions are shown in Table 1 , the units being logarithms to base 10 of the concentrations in particles $/ \mathrm{ml}$.

These standard deviations are very similar, but by means of a test due to Bartlett (1937) it can in fact be shown that the three estimates differ more than would be expected by chance alone. Nonetheless, the differences are small and the pooled
TABLE 1

ANALYSIS OF THE VARIATION OF CONCENTRATIONS WITHIN A SHIFT OBSERVED IN THREE RANDOMCOLLIER SURVEYS

\begin{tabular}{c|c|c|c|c}
\hline Survey & $\begin{array}{c}\text { Sum of Squared } \\
\text { Deviations from } \\
\text { Shift Mean }\end{array}$ & $\begin{array}{c}\text { Degrees } \\
\text { of } \\
\text { Freedom }\end{array}$ & Variance & $\begin{array}{c}\text { Standard } \\
\text { Deviation }\end{array}$ \\
\hline A & 11.990458 & 303 & $0 \cdot 039572$ & $0 \cdot 1989$ \\
B & $16 \cdot 604184$ & 270 & $0 \cdot 061497$ & $0 \cdot 2480$ \\
B & $9 \cdot 059755$ & 203 & 0.044629 & $0 \cdot 2113$ \\
Pooled & 37.654397 & 776 & 0.048524 & $0 \cdot 2203$ \\
\hline
\end{tabular}

The units are logarithms to base 10 of concentrations in particles $/ \mathrm{ml}$. 


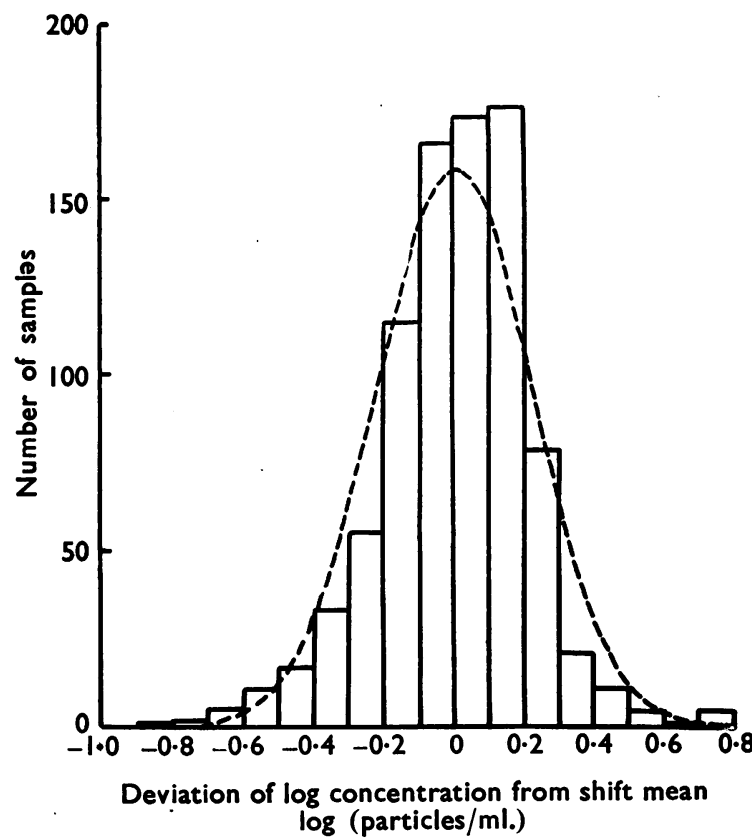

FIG. 2.-Distribution of deviations of 779 transformed samples from their shift means, with the Normal curve of equal area and standard deviation $\mathbf{0 . 2 2 0 3}$.

curve of standard deviation $0 \cdot 2203$ and of equal area is also shown. The fit is fair, but evidently the logarithmic transformation somewhat over-corrects the skewness of the original data, which are also more closely concentrated around their mean than is the Normal curve*. In the normal curve, $5 \%$ or 43.7 results should fall outside the limits -0.432 to 0.432 . In fact, 44 are found outside these limits, though unevenly placed, 15 on the positive side, 29 on the negative.

Figure 3 shows the cumulative distribution curve of the transformed data and (broken curve) of the fitted normal curve. The abscissae are deviations, in standard deviation units, of the transformed data from the log geometric mean (the arithmetic mean of the logarithms). It will be seen that the normal curve overestimates the proportion of samples found to exceed the geometric mean except at very large deviations, but is never incorrect by more than $5 \%$.

In practical work, it appears that the assumption that the logarithms of dust concentrations on a face vary in accordance with a Normal law of constant

* The standard deviation of the Normal curve has been estimated from a number of small sets of samples; the divisor used was therefo-e the total number of samples less the number of sets of samples. This gives the best estimate of the population standard deviation, but it will be, on the averag
each small se tof samples. standard deviation will not lead us far astray. We may safely use for such transformed data the statistical devices, such as standard errors, appropriate to normally distributed variates. For the recording of averages, however, the transformation is unnecessary. The simple arithmetic mean of a set of samples is an unbiased estimate of the true mean concentration even if the distribution is lognormal ; that is, in the long run, the means of such sets will be scattered evenly above and below the true mean, and their mean will tend ever closer to it as the number of sets of samples increases. It can be shown, however, that the scatter of these means will be larger than that of some other estimates derived from the samples. They are in fact inefficient estimators, which do not make full use of the information given by the samples about the true mean. In some situations the excessive scatter of inefficient estimators can be a serious source of loss. It was observed by Sichel (1952) that the distribution of richness of ore in gold mines was lognormal, with a large standard deviation, and that in consequence if costly errors in interpreting samples of ore were to be avoided, it was essential to use estimators other than the arithmetic mean of the samples. However, the inefficiency of the arithmetic mean in a distribution with such a standard deviation as we have found is too small to be of any practical importance. Finney (1941) shows that the loss of information in this case will amount to no more than $1 \%$.

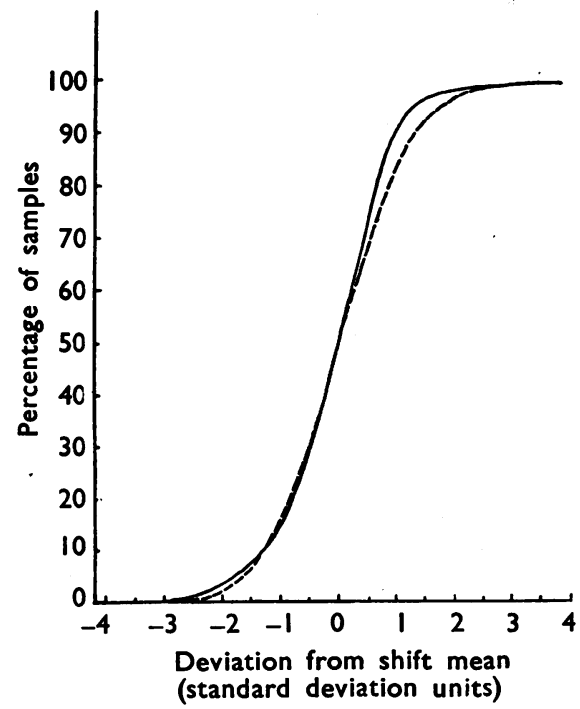

Fig. 3.-Cumulative distribution of deviations of 779 transformed samples from their shift means (full line) and of the fitted Normal curve (broken line). 
Finney's results are, however, necessary in order to find the relationship between the arithmetic and geometric means in samples from a lognormal population to enable us to interpret the results of statistical calculations, such as of the percentile points of the distribution, necessarily made with the logarithms of sampling data. Such results would be expressed in terms of geometric mean concentrations, and would need to be converted into terms of the ordinary arithmetic mean.

Finney shows that an efficient and unbiased estimator of the arithmetic means of a lognormal population is obtained by multiplying the geometric mean of the sample of $n$ by a function $g\left(\frac{1}{2} s^{2}\right)$, where $\mathbf{s}^{2}$ is the usual unbiased estimator of the population variance derived from the sample, $\Sigma(\mathrm{x}-\overline{\mathrm{x}})^{2} /(\mathrm{n}-1)$, the $x$ s being the natural logarithms of the sample concentrations, and $g(t)$ can be expanded as the series

$1+\frac{n-1}{n} \cdot t+\frac{(n-1)^{3}}{n^{2} \cdot 2 !} \cdot \frac{t^{2}}{n+1}+\frac{(n-1)^{5}}{n^{3} \cdot 3 !} \cdot \frac{t^{3}}{(n+1)(n+3)}+\ldots$

By simple extension of his results it can be shown that when, as in our case, the variance has previously been estimated with a large number of degrees of freedom, an efficient estimator of the arithmetic mean is given by the geometric mean of n samples multiplied by $\mathrm{g}\left\{(\mathrm{n}-1)(\mathrm{m}+1) \mathrm{s}^{2} / 2 \mathrm{mn}\right\}$ where $m$ is the number of degrees of freedom upon which the estimate $\mathrm{s}^{2}$ is based. In our case $\mathrm{m}=776$, and using $s=0.2203 \times \log _{\mathrm{e}} 10$, the multiplier becomes

$$
\begin{aligned}
& 1+0.128634(n-1) / n+0.008252\{(n-1) / n\}^{2} \\
+ & 0.000352\{(n-1) / n\}^{3}+0.000010\{(n-1) / n\}^{4}+\ldots
\end{aligned}
$$

The values of this function are shown in Fig. 4 for different values of $n$, the number of samples per

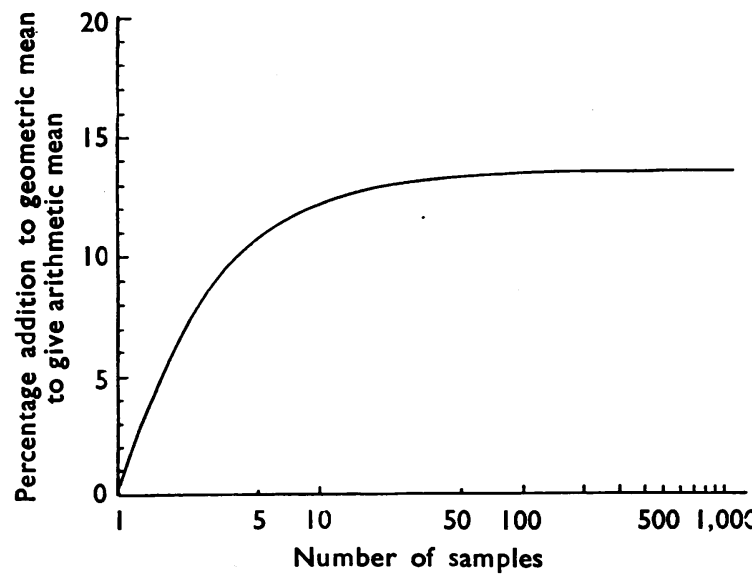

FIG. 4.-Relationship between estimated geometric mean and arithmetic mean for different numbers of samples.
TABLE 2

PERCENTILE POINTS OF DISTRIBUTION OF CONCENTRATIONS WITHIN A SHIFT *

\begin{tabular}{c|c|c}
\hline Percentile & Lognormal Curve & Experimental Curve \\
\hline 1 & 27.3 & 21.8 \\
5 & 38.7 & 36.3 \\
10 & 46.5 & 46.2 \\
20 & 58.1 & 59.8 \\
30 & 68.2 & 69.7 \\
50 & 89.3 & 88.0 \\
70 & 116.1 & 110.3 \\
80 & 136.4 & 123.7 \\
90 & 170.5 & 142.7 \\
95 & 205.0 & 165.7 \\
99 & 290.0 & 288.0 \\
\hline
\end{tabular}

- Percentile points are expressed as percentages of the mean concentration; e.g., $10 \%$ of concentrations would be expected to be less than $46 \%$ of the mean concentration, while $1 \%$ would exceed $290 \%$ of the mean.

day, in the form of the percentage addition to be made to the geometric mean of the sample.

For large $n$, the addition stabilizes at $13.72 \%$, and over the range from 6 to 12 , in which most of our sets of samples lie, the value of $12 \%$ is an adequate approximation. If we assume, therefore, that the arithmetic mean of our samples is on the average $112 \%$ of the geometric mean, we may then convert the percentile points of the distribution of observations, which have been obtained as percentages of the geometric mean, to percentages of the arithmetic mean by multiplying each by the reciprocal of $112 \%$, that is, $89 \%$.

The percentile points of the lognormal curve and of the experimental curve are shown in Table 2. Reference to Fig. 1 suggests that the percentile points of the whole set of results fall somewhat closer to the lognormal curve than to that derived from the random collier results. The lognormal curve remains, as was suggested, a good approximation to the truth.

\section{Discussion}

Although the variation of dust concentrations at the coal-face during a single shift is great, it now appears that it is nonetheless regular and predictable, and, to a very great extent, closely related to the average concentration. It is necessary to consider if this finding really represents a property of the dust conditions, or if it might be, to a greater or lesser extent, an artifact produced by the sampling instrument or method. It is difficult to conceive of anything in the sampling methods which could cause an artificial regularity of variation. The sampling instrument used, the thermal precipitator, produces a deposit of dust upon two cover-glasses, a small fraction of which (about $1 \%$ ) is subsequently examined and counted under the microscope. The deposit is not uniform, but shows systematic changes from place to place. If it were 


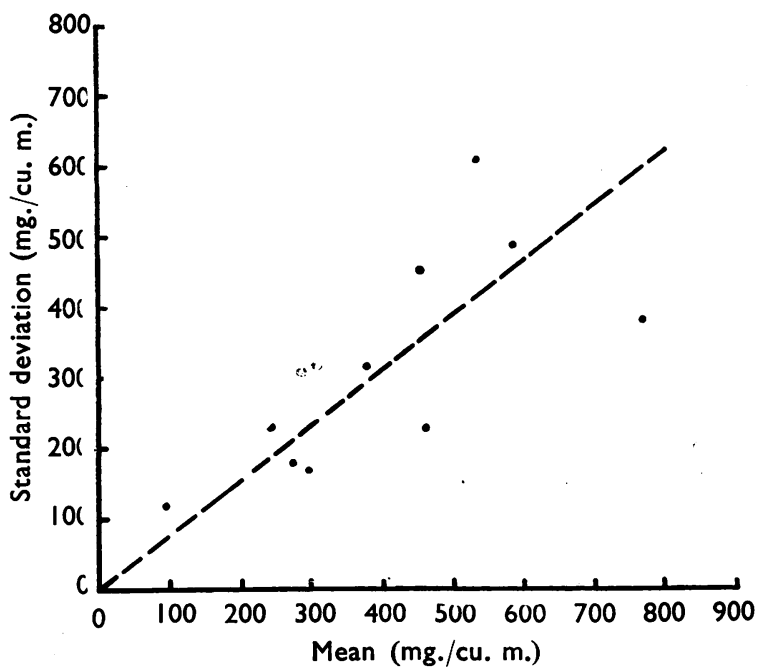

FIG. 5.-Relationship between mean and standard deviation in gravimetric sampling results given by Briscoe and others (1943).

uniform, it is known that an error due to random variation in the deposit must result since only a fraction is counted. In addition, the count will be subject to observer error. These errors will be of a kind which would increase with dust concentration. However, from unpublished results obtained at this Unit 'it has been estimated that the overall effect of these factors is to produce a standard error of about 5 to $10 \%$ of the count, a source of variation considerably smaller than that found. This standard error must be included in the apparent variation, and may be in part the cause of the observed deviations from an exactly lognormal distribution, but its relative importance is small.

The standard deviation in units of log (particles/ $\mathrm{ml}$.) has been estimated as 0.2203 ; its antilogarithm, 1.66 , may be interpreted as a proportional standard deviation of $66 \%$ about the geometric mean. This of course is not the same figure as would be obtained by expressing the standard deviation of the untransformed observations as a percentage of their mean (the coefficient of variation). The latter figure could be most misleading as a basis for calculating standard errors in view of the non-normality of the distribution and, as Finney (1941) points out, is a most inefficient statistic for estimating the true standard deviation of the distribution, equivalent, if we assume an exactly lognormal distribution, to discarding $35 \%$ of the data. From Finney's results we may deduce an efficient estimate of the standard deviation to be the geornetric mean multiplied by the square root of $\mathrm{g}\left\{2(\mathrm{~m}+1)(\mathrm{n}-1) \mathrm{s}^{2} / \mathrm{mn}\right\}-\mathrm{g}\left\{(\mathrm{m}+1)(\mathrm{n}-2) \mathrm{s}^{2} / \mathrm{mn}\right\}$ where $\mathbf{g}$ is the function defined above and $\mathrm{m}, \mathrm{n}$, and $s$ have the same meaning as before. This function has a similar form to that shown in Fig. 4, and with the value we have found for $\mathrm{s}$ and $\mathrm{m}$ equal to 776 it increases from 0.542 when $n=2$ to 0.616 when $\mathrm{n}$ is infinite. Over the range from $\mathrm{n}=6$ to 12 the value 0.60 is a close approximation. Thus the standard deviation of untransformed samples would be expected to be $0.89 \times 0.60$ of their arithmetic mean, or $53 \%$.

The only estimates of the standard deviations of thermal precipitator samples that have been found in the literature are those given by Bedford and Warner (1943). Their estimates for five collieries range from 40 to $60 \%$ of the mean, averaging $44.7 \%$ if samples taken after shot-firing are excluded, $48.6 \%$ if they are not. It can be shown that this simple average of the coefficients of variation always gives an underestimate of the true value. A better figure, though still a slight underestimate, is their root mean square. In this case the difference is small, the root mean square estimates being $45 \%$ and $50.3 \%$ respectively. In view of the fact that their samples were usually taken over 20 minutes, and so should vary less than ours, but probably extended for more than one shift, and so included changes in the level of the shift mean, little can be deduced from the comparison except that the order of magnitude is correct.

The distribution of mass concentration of total airborne dust given by Briscoe, Holt, Spoor, Matthews, and Sanderson (1943) provides further evidence that the lognormal distribution of concentrations is real and not artificial. From their Table XLII it may be seen that the distribution of mass concentrations was skewed in the way expected of a lognormal distribution. From their Table XL the standard deviations for each set of data have been calculated (by multiplying the standard errors quoted by the square root of the number of observations) and these are compared with the given average concentrations in Fig. 5. It will be seen that the standard deviation increases with the mean, averaging about $78 \%$.of it. The root mean square is $88 \%$. It would be expected that mass concentrations of total dust would be more variable than number concentrations, since the presence or absence of a few large particles would make substantial changes in the total mass.

Further evidence is provided by a personal communication from Mr. D. Young, who has informed me that in his routine dust sampling in the copper mines of $\mathrm{N}$. Rhodesia, in which the sampling instrument was a konimeter, he has observed that the proportion of samples exceeding the mean by given multiples remains constant, 
although the mean level of concentration has been steadily diminishing during the past few years.

It appears justifiable to assume that the lognormal law of constant proportional standard deviation that has been observed in the present data is a property of underground dust conditions that would appear whatever the sampling instrument used. Certain deductions of a practical nature can at once be made. In the first place, the accuracy of the mean concentration during a shift estimated by thermal precipitator sampling can be specified in advance. The standard deviation of $20 \mathrm{ml}$. samples in units of $\log$ (particles $/ \mathrm{ml}$.) has been

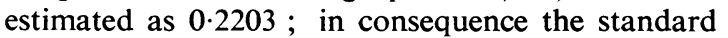
error of the mean of $n$ such samples will be $0 \cdot 2203^{\prime} \sqrt{ } \mathrm{n}$ and $95 \%$ fiducial limits to the mean will be given by $\mathrm{m} \pm 0.4318 / \sqrt{ } \mathrm{n}$. Taking antilogarithms, and remembering that the geometric and arithmetic means will be practically proportional, we may express the accuracy of average concentrations for the shift in terms of proportional limits, as in Fig. 6. For example, a set of $1020-\mathrm{ml}$. thermal precipitator samples will enable us to state that there is but one chance in twenty of getting the observed mean if the true shift mean lies outside the range of $73 \%$ to $137 \%$ of the observed mean. Even if 50 samples are taken, the limits reduce only to $87 \%$ and $115 \%$, a range of some $23 \%$ of the calculated mean.

Only if sampling were continuous throughout the shift would this range of uncertainty disappear. With an automatic dust-sampling instrument such continuous sampling would fall within the bounds of practical possibility, and the need for such an instrument is made even clearer when it is remembered that the ultimate purpose of all dust sampling in coal-mines is to estimate what dust may be inhaled by coal miners as a result of mining operations. Pneumoconiosis appears very slowly; the contribution of a single day's exposure must be minute. It is clearly necessary to measure and control the dust conditions by means of sampling throughout an extended series of days. If sufficient samples are taken by means of a hand-operated instrument to give a reasonably accurate estimate of a single day's concentration the number required to cover a longer period becomes prohibitive. If on the other hand only a few samples are taken each day but many days are covered, random fluctuations at least as great as those revealed in the present data will appear in the daily averages. By means of a system of quality control, such as is used to monitor the output of industrial processes, the results could be satisfactorily utilized, but the cost of the method would be excessive. An automatic sampling instrument is the only economical solution.

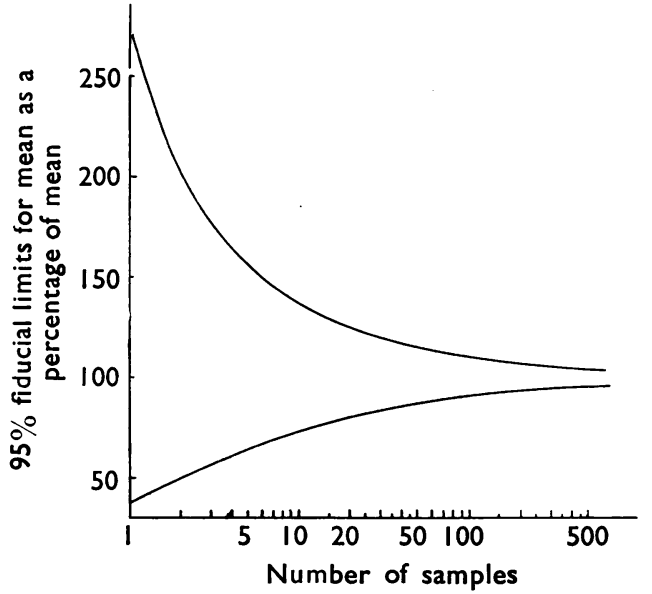

FIG. 6.-Ninety-five per cent. fiducial limits for the mean concentration obtained from different numbers of thermal precipitator samples.

Finally, it is of some interest to consider if any light is thrown on the nature and consequences of "approved conditions" by the present findings. Approved conditions are expressed in terms of the permissible concentration of airborne dust, the standard for non-anthracite collieries being " not more than 850 particles per c.c. between 1.0 and 5.0 microns in size". The National Coal Board circular CD/354 (1948) remarks that "The standards are not meant to be peak measurements. They are meant to show the conditions as determined by several measurements made at representative times and points. The measurements from which the averages are calculated are, therefore, not to be made in abnormal conditions." Subsequently, another circular (National Coal Board, 1949) gives detailed proposals for the method of sampling to be adopted. This states that "the standards are to be interpreted not as defining the allowable average concentration of dust over the whole period of a working shift but the average dustiness during periods of maximum dust production". It is made clear that these periods are not those of momentary peak concentrations, but rather of whole sections of the working shift. On the filling-off shift the first four to four and a half hours are suggested as being the period of maximum dust production, but any exceptionally dusty spell of, say, one and a half hours during this period should be considered separately.

Evidently it is not possible to determine from these rules alone what connexion there will be between the overall average of the shift and the average of the " period of maximum dust production". It is, 
however, the former figure which, in conjunction with the time over which it holds, determines the dose of dust which the colliers receive, and the connexion between the two is thus of great practical interest. The practical interpretation of the administrative instructions quoted above has come to be (Hicks, 1953) that conditions are taken as being " approved" if $90 \%$ of samples taken when work is in progress on the face do not exceed 850 particles/ $\mathrm{ml}$. We may thus refer this criterion to the results we have presented here. The work of Wynn and Dawes (1951) shows that within the microscopic size range the size distribution of airborne dust is remarkably constant. Thus although the concentration of 850 particles $/ \mathrm{ml}$. is specified to relate to dust ${ }^{*}$ between $1 \mu$ and $5 \mu$, a constant factor of proportionality will convert this to the range we have used, $0.5 \mu$ to $5 \mu$, and this descrepancy may be ignored. The proportional relationship between the mean and the $90 \%$ point will be the same whichever range is used.

Fig. 3 shows that if 850 particles $/ \mathrm{ml}$. corresponds to the $90 \%$ point of the curve, the geometric mean will fall 1.28 standard deviation units below if the normal curve is used, 0.88 standard deviation units below if the experimental curve is used. The corresponding geometric mean concentrations are 444 or 544 , and, multiplying by $112 \%$, the arithmetic means are 500 or 610 . It is unfortunate that the discrepancy between the two curves is at its greatest at the $90 \%$ point, but we can be reasonably sure that this interpretation of approved conditions is equivalent to an average concentration of between 500 and 600 particles $/ \mathrm{ml}$. during the working shift, irrespective of the level of activity on the face. Conversely, given an average concentration between these limits, we may be confident that in the long run peaks of concentration exceeding 850 particles/ $\mathrm{ml}$. will occur regularly during $10 \%$ of the working time. In fact, if it be maintained (despite the lack of any evidence to that effect) that it is solely such "peaks" that constitute a serious risk to health, no difference in sampling procedure is required. A technique which estimates the average level of concentration and, equally important, the duration of exposure, at the same time estimates the exposure to peak concentrations, since their frequency can at once be deduced.

\section{Summary}

Dust samples taken with the thermal precipitator at the coalface by the Pneumoconiosis Research
Unit are analysed to show the kind of variation in dust concentration that is experienced.

Although the variability is high, the general run of samples suggests that the range of concentrations is considerably dependent on the average level, and agrees quite well with the assumption of a lognormal distribution of concentrations with an almost constant proportional standard deviation.

In consequence the accuracy to be expected in estimating mean concentrations from numbers of thermal precipitator samples can be specified in advance. The $95 \%$ fiducial limits of the mean of 10 such samples are $73 \%$ to $137 \%$, a range equal to $64 \%$ of the calculated mean. Even 50 samples only reduce this range to $23 \%$.

The necessity is pointed out of automatic sampling instruments, which alone can sample inexpensively for sufficiently long to reduce this sampling error, and which are demanded also from consideration of the aetiology and rate of appearance of pneumoconiosis.

On the assumption that "approved conditions" are such that $90 \%$ of dust concentrations during a shift are less than 850 particles $/ \mathrm{ml}$., it is deduced that this limit is equivalent to an average concentration of between 500 and 600 particles $/ \mathrm{ml}$. throughout the working shift.

I am grateful for the helpful advice I have received from my colleagues in the Pneumoconiosis Research Unit, in particular, from Mr. R. G. Carpenter and Mr. S. A. Roach. Mr. Roach in addition provided the data upon which the work is based. The diagrams were prepared by Mr. F. Meade.

\section{REFERENCES}

Bartlett, M. S. (1937). J. roy. statist. Soc., Suppl., 4, 137.

Bedford, T., and Warner, C. G. (1943). Spec. Rep. Ser., med. Res. Counc., Lond., No. 244, p. 53.

Briscoe, H. V. A., Holt, P. F., Spoor, N., Matthews, J. W., and Sanderson, P. M. (1943).' Ibid., No.'244, p. 86 .

Finney, D. J. (1941). J. roy. statist. Soc., Suppl., 7, 155.

Hicks, D. (1953). Personal communication.

Long, W. M. (1953). British Journal of Industrial Medicine, 10, 241.

National Coal Board (1948). Circular CD/354: Employment of Pneumoconiosis Cases.

(1949). Circular: The Sampling of Air-borne Dust for the Testing of "Approved Conditions".

Oldham, P. D., and Roach, S. A. (1952). British Journal of Industrial Medicine, 9, 112.

Sichel, H. S. (1952). Trans. Instn Min. Metall., Lond., 61, 261.

Watson, H. H. (1949). Proc. 9th Int. Congr. Industr. Med. London, 1948, p. 1029. Wright, Bristol.

Wright, B. M. (1953). British Journal of Industrial Medicine, 10, 235.

Wynn, A. H. A., and Dawes, J. G. (1951). Ministry of Fuel and No. 28. The Size Classification of Airborne Dusts in Mines. 